\title{
Future directions of the Virtual Observatory
}

\section{Alexander S. Szalay}

Physics and Astronomy Department, Johns Hopkins University, Charles and 34th St, Baltimore, MD 21218-2686, USA

email: szalay@jhu.edu

The Virtual Observatory effort has undergone a substantial evolution over the last four years. In the talk we will discuss the directions of the current effort, and consider the direction along which the Virtual Observatory might evolve along with various other large astronomy projects. We will also discuss similar efforts in other disciplines and the relation of the VO to Grid Computing. 